\title{
Unconditional security proof of a deterministic quantum key distribution with a two-way quantum channel
}

\author{
Hua Lu, ${ }^{1,2}$ Chi-Hang Fred Fung, ${ }^{3}$ Xiongfeng Ma, ${ }^{4}$ and Qing-yu Cai ${ }^{1, *}$ \\ ${ }^{1}$ State Key Laboratory of Magnetics Resonances and Atomic and Molecular Physics, Wuhan Institute of Physics and Mathematics, \\ Chinese Academy of Sciences, Wuhan 430071, People's Republic of China \\ ${ }^{2}$ Department of Mathematics and Physics, Hubei University of Technology, Wuhan 430068, People's Republic of China \\ ${ }^{3}$ Department of Physics and Center of Computational and Theoretical Physics, University of Hong Kong, Pokfulam Road, Hong Kong \\ ${ }^{4}$ Center for Quantum Information and Quantum Control, Department of Physics, University of Toronto, Toronto, Canada, M5S 1A7
}

(Received 13 June 2011; published 31 October 2011)

\begin{abstract}
In a deterministic quantum key distribution (DQKD) protocol with a two-way quantum channel, Bob sends a qubit to Alice who then encodes a key bit onto the qubit and sends it back to Bob. After measuring the returned qubit, Bob can obtain Alice's key bit immediately, without basis reconciliation. Since an eavesdropper may attack the qubits traveling on either the Bob-Alice channel or the Alice-Bob channel, the security analysis of DQKD protocol with a two-way quantum channel is complicated and its unconditional security has been controversial. This paper presents a security proof of a single-photon four-state DQKD protocol against general attacks.
\end{abstract}

DOI: 10.1103/PhysRevA.84.042344

PACS number(s): 03.67.Dd

\section{INTRODUCTION}

Quantum cryptography enables two remote parties to share an information-theoretically secure key, which can be used for later cryptographic applications. Since the pioneering protocol was presented by Bennett and Brassard in 1984 (called the BB84 protocol) [1], its security against general attacks has been studied in idealized settings [2-4] and in practical settings [5]. Meanwhile, a deterministic quantum key distribution (DQKD) protocol with a two-way quantum channel has been proposed [6,7], which allows Alice and Bob to encode and decode secret messages in a deterministic manner. Bob can obtain Alice's key bit directly with his measurement outcomes, without a basis reconciliation step, which makes key distribution efficient and even a quasisecure direct communication possible when the two parties are connected with an ideal two-way quantum channel $[6,8]$. As for experimental demonstrations, DQKD protocols without entanglement, e.g., the single-photon two-state $\mathrm{DQKD}$ protcol [9] and the single-photon four-state DQKD protocol [10] (we call it the four-state protocol hereafter), were proposed. Although the security of the four-state protocol against some special individual attacks has been considered [11], its security against general attacks has not been proved [12].

The difficulty of the security proof for the DQKD protocol against general attacks is due to the use of a two-way quantum channel. In the BB84 protocol, a qubit just travels from Alice to Bob once, carrying one-bit secret information. Upon receiving the qubits, Bob measures it in either of the alternative bases to obtain Alice's key bits. A powerful eavesdropper, Eve, whose capacity is only limited by the physical laws, may attack the information-carrying qubit in the one-way quantum channel. In the DQKD protocol [6], however, a qubit departs from Bob to Alice (the forward channel, Bob-Alice), and then it carries Alice's secret key bits back to Bob (the backward channel, Alice-Bob). In this case, Eve might attack the qubits traveling on both the Bob-Alice channel and the Alice-Bob channel.

*qycai@wipm.ac.cn
Comparing it with the BB84 protocol, the security analysis of the DQKD protocol is complicated and its unconditional security has not been proved before [12]. In fact, the security of the two-way DQKD protocol has been challenged over time (see, e.g., [7,13-15]). Some of these challenges have led to refinement of the protocol and some have been refuted [12]. In this paper, we present a security proof of the four-state protocol against general attacks, thus confirming the unconditional security of the protocol.

This paper is organized as follows. In Sec. II, we introduce the four-state protocol. Next in Sec. III, we present the security proof and final key generation rate against collective attacks. Then we extend our security proof and key generation against general attacks. We finally conclude in Sec. IV.

\section{FOUR-STATE PROTOCOL}

The four-state protocol works as follows:

(1) Bob prepares $n$ qubits randomly in one of the four states, $|0\rangle,|1\rangle,|+\rangle$, and $|-\rangle$, where $| \pm\rangle=(|0\rangle \pm|1\rangle) / \sqrt{2}$ and sends them to Alice.

(2) In the check mode, Alice randomly measures part of the received states in the $X$ or $Z$ basis.

(3) In the encoding mode, Alice randomly performs the unitary operations $I=|0\rangle\langle 0|+| 1\rangle\langle 1|$ (bit 0 ) or $Y=|0\rangle\langle 1|-$ $|1\rangle\langle 0|$ (bit 1) on the rest received states.

(4) Alice sends the encoded qubits back to Bob. It is interesting to note that $Y\{|0\rangle,|1\rangle\}=\{-|1\rangle,|0\rangle\}$, and $Y\{|+\rangle,|-\rangle\}=$ $\{|-\rangle,-|+\rangle\}$, so Bob measures each qubit in the same basis as the one he used for preparation. In this way, Bob can obtain Alice's key bits deterministically, without basis reconciliation [9-11].

(5) After Bob measures all returned qubits, Alice announces her measurement results in the check mode. They compute the fidelity of the forward states with results of consistent-basis measurements, i.e., Alice measures the forward state in the same basis as Bob's preparing it. For instance, when Bob sends a state $|0\rangle$ and Alice measures it in the $Z$ basis in check mode, with a probability of $f_{0}$, Alice's measurement outcome is $|0\rangle$. 
Similarly, they can calculate the fidelity $f_{1}, f_{+}$, and $f_{-}$of $|1\rangle$, $|+\rangle$, and $|-\rangle$, respectively. Alice and Bob discard results from the inconsistent-basis measurements.

(6) Alice announces partial of her key bits in the encoding mode. They compute the error rate $e$ in the Alice-Bob channel.

(7) If Alice and Bob find the error rates in the Bob-Alice channel are not too high [satisfying Eq. (9)], they will continue the protocol, i.e., Alice and Bob will perform error correction (EC) and privacy amplification (PA) to gain the secure final key bits. Otherwise, we assume Alice and Bob will abort the protocol.

\section{SECURITY ANALYSIS}

\section{A. Eve's attack in the Alice-Bob channel}

Suppose that Eve only attacks the qubits in the Alice-Bob channel. Bob prepares the forward qubits randomly in the state $|0\rangle,|1\rangle,|+\rangle$, and $|-\rangle$ with the same probability, i.e., the forward qubit is prepared in a mixed state, $\rho^{B}=(|0\rangle\langle 0|+$ $|1\rangle\langle 1|+|+\rangle\langle+|+|-\rangle\langle-|) / 4=(|0\rangle\langle 0|+| 1\rangle\langle 1|) / 2$. To gain Alice's key bits information, Eve has to distinguish Alice's encoded qubit $\rho_{0}^{B}=I \rho^{B} I$ from $\rho_{1}^{B}=Y \rho^{B} Y$ in the Alice-Bob channel. Since $\rho_{0}^{B}=\rho_{1}^{B}=(|0\rangle\langle 0|+| 1\rangle\langle 1|) / 2$, Eve cannot gain any information about Alice's key bits if she only attacks the qubits after Alice's encoding operation. Therefore, we can conclude that Eve has to attack the qubits traveling on both the Bob-Alice channel and the Alice-Bob channel in order to gain Alice's key bits.

\section{B. Eve's attack in the Bob-Alice channel}

Eve's most general quantum operation can be described by a unitary operation together with an ancilla [16]. In the Bob-Alice channel, when Bob sends a qubit in state $|0\rangle$ and Alice measures in the basis $|0\rangle,|1\rangle$, she will get the measurement outcomes $|0\rangle$ with probability $c_{00}^{2}$, or $|1\rangle$ with probability $c_{01}^{2}$. Define $f_{0}=c_{00}^{2}$ as the fidelity of state $|0\rangle$, which can be verified by Alice and Bob in their postprocessing. Similarly, Alice and Bob can obtain $f_{1}=c_{11}^{2}, f_{+}=c_{++}^{2}$, and $f_{-}=c_{--}^{2}$, respectively. Since the state space of the forward qubit is two dimensional, Eve's most general attack in the Bob-Alice channel can be written in the form

$$
\begin{aligned}
& U_{B E}|0\rangle_{B}|E\rangle=c_{00}|0\rangle_{B}\left|E_{00}\right\rangle+c_{01}|1\rangle_{B}\left|E_{01}\right\rangle, \\
& U_{B E}|1\rangle_{B}|E\rangle=c_{11}|1\rangle_{B}\left|E_{11}\right\rangle+c_{10}|0\rangle_{B}\left|E_{10}\right\rangle,
\end{aligned}
$$

and

$$
\begin{aligned}
& U_{B E}|+\rangle_{B}|E\rangle=c_{++}|+\rangle_{B}\left|E_{++}\right\rangle+c_{+-}|-\rangle_{B}\left|E_{+-}\right\rangle, \\
& U_{B E}|-\rangle_{B}|E\rangle=c_{--}|-\rangle_{B}\left|E_{--}\right\rangle+c_{-+}|+\rangle_{B}\left|E_{-+}\right\rangle,
\end{aligned}
$$

where $c_{i j}, c_{++}, c_{+-}, c_{--}$, and $c_{-+}$can be treated as nonnegative real numbers, ${ }^{1}$ and $\left|E_{i j}\right\rangle,\left|E_{++}\right\rangle,\left|E_{+-}\right\rangle,\left|E_{--}\right\rangle$, and

\footnotetext{
${ }^{1}$ In general, the coefficients are a complex number and $\left|E_{i j}\right\rangle$ are normalized vectors. For simplicity, we can rewrite the formula where the phase of each coefficient is absorbed into the companying. For instance, $c_{i j}\left|E_{i j}\right\rangle=\sqrt{c_{i j} c_{i j}^{*}} e^{i \delta_{i j}}\left|E_{i j}\right\rangle=\sqrt{c_{i j} c_{i j}^{*}}\left(e^{i \delta_{i j}}\left|E_{i j}\right\rangle\right)$. Thus, we can get that $c_{i j} \equiv \sqrt{c_{i j} c_{i j}^{*}}$ are non-negative real numbers and $\left|E_{i j}\right\rangle \equiv$ $e^{i \delta_{i j}}\left|E_{i j}\right\rangle$ are normalized vectors.
}

$\left|E_{-+}\right\rangle$forms four pairs of normalized vectors. For now, we consider the case that Eve performs a collective attack, i.e., $U_{B E}$ are the same for all qubits. This restriction can be removed with the quantum de Finetti theorem [17-19], and then we can prove the four-state protocol is secure against general attacks.

As discussed above, Bob's forward qubit is prepared in a mixed state $\rho^{B}=(|0\rangle\langle 0|+| 1\rangle\langle 1|) / 2$. After Eve's attack in the Bob-Alice channel, the joint state of the forward qubit and Eve's ancilla becomes

$$
\rho_{\text {Bob-Alice }}^{B E}=U_{B E}\left(\rho^{B} \otimes|E\rangle\langle E|\right) U_{B E} .
$$

After receiving the forward qubits, in the encoding mode, Alice will encode her key bits onto the forward qubit. With probability $p=1 / 2$, she encodes key bit 0 by the operation $I_{B}$ or key bit 1 by the operation $Y_{B}$. After the encoding, the state of the qubit and Eve's ancilla becomes

$$
\rho^{A B E}=\frac{1}{2}|0\rangle\left\langle\left. 0\right|^{A} \otimes \rho_{0}^{B E}+\frac{1}{2} \mid 1\right\rangle\left\langle\left. 1\right|^{A} \otimes \rho_{1}^{B E},\right.
$$

where $\rho_{0}^{B E}=\rho_{\text {Bob-Alice }}^{B E}$, and $\rho_{1}^{B E}=Y_{B} \rho_{\text {Bob-Alice }}^{B E} Y_{B}$. Next, Alice sends the encoded qubits back to Bob.

After Bob measured all the returned qubits, Alice will announce her measurement outcomes in the check mode, so that they can gain the fidelity of Bob's forward states, $f_{0}, f_{1}$, $f_{+}$, and $f_{-}$in the Bob-Alice channel. Alice will also publish some of her key bits to gain the error rate $e$ of the key bits in the Alice-Bob channel. For simplicity, we will consider first the case that $f_{0}=f_{1}$ and $f_{+}=f_{-}$.

\section{Secret key generation}

The asymptotic key generation rate can be defined as $r=\lim _{m \rightarrow \infty} k(m) / m$, where $m$ is the size of the raw key and $k(m)$ is the number of the final key bits. Alice sends Bob EC information over a classical channel so that he can correct his raw key to match Alice's. This EC information is encrypted using pre-shared secret key bits and thus is unknown to Eve. The final key is then derived by applying two-universal hashing to their common raw key as PA [20]. In the asymptotic scenario, the secure key rate $r_{\mathrm{PA}}$ for secret key generation is bounded by the conditional entropy of Alice and Bob's key bits given the quantum information of Eve about the key bits, $r_{\mathrm{PA}}=S\left(\rho^{A} \mid \rho^{B E}\right)$.

After Alice's encoding operations, Eve can gain some quantum information about Alice's key bit from the quantum state $\rho^{B E}=\operatorname{tr}_{A} \rho^{A B E}$ that is a joint state of the backward qubit and her ancilla. Here, we assume the worst case that Eve uses the entire state $\rho_{B E}$ to gain information about the key bit, even though she may have to send part of the state to Bob. With Renner and König's results [20], we have $r_{\mathrm{PA}}=S\left(\rho^{A} \mid \rho^{B E}\right)=$ $S\left(\rho^{A B E}\right)-S\left(\rho^{B E}\right)$, where $S\left(\rho^{A B E}\right)=-\operatorname{tr} \rho^{A B E} \log _{2} \rho^{A B E}$, and $S\left(\rho^{B E}\right)=-\operatorname{tr} \rho^{B E} \log _{2} \rho^{B E}$. In the following, we should calculate the eigenvalues of $\rho^{A B E}$ and $\rho^{B E}$ to get $S\left(\rho^{A B E}\right)$ and $S\left(\rho^{B E}\right)$.

\section{Key generation rate for PA}

Let us denote that

$$
\begin{aligned}
& \left\langle E_{00} \mid E_{01}\right\rangle=s_{0}+i s_{1}, \\
& \left\langle E_{00} \mid E_{10}\right\rangle=u_{0}+i u_{1},
\end{aligned}
$$




$$
\begin{aligned}
\left\langle E_{00} \mid E_{11}\right\rangle & =p_{0}+i p_{1}, \\
\left\langle E_{11} \mid E_{10}\right\rangle & =r_{0}+i r_{1}, \\
\left\langle E_{01} \mid E_{11}\right\rangle & =v_{0}+i v_{1}, \\
\left\langle E_{01} \mid E_{10}\right\rangle & =q_{0}+i q_{1},
\end{aligned}
$$

where $p_{i}, q_{i}, r_{i}, s_{i}, u_{i}$, and $v_{i}$ are real numbers. Taking the inner product of two equations of Eq.(1) gives

$$
c_{00} c_{10}\left\langle E_{10} \mid E_{00}\right\rangle+c_{11} c_{01}\left\langle E_{01} \mid E_{11}\right\rangle=0 .
$$

For simplicity, we rewrite $c_{0} \equiv c_{00}=c_{11}$ and $c_{1} \equiv c_{01}=c_{10}$ and thus we have $u_{0}=-v_{0}$ and $u_{1}=-v_{1}$.

Let us calculate $S\left(\rho^{A B E}\right)$ and $S\left(\rho^{B E}\right)$. A straightforward computation $^{2}$ shows that the eigenvalues of $\rho^{A B E}$ can be obtained as $\lambda_{0,1,2,3,4,5,6,7,8,9,10,11}^{A B E}=0$ and $\lambda_{12,13,14,15}^{A B E}=1 / 4$. Thus, we obtain $S\left(\rho^{A B E}\right)=\sum_{i} \lambda_{i}^{A B E} \log _{2} \lambda_{i}^{A B E}=2$, where we have used the convention $0 \log _{2} 0=0$. The eigenvalues of $\rho^{B E}=\operatorname{tr}_{A} \rho^{A B E}$ are $\lambda_{0,1,2,3}^{B E}=0$, $\lambda_{4}^{B E}=\frac{1}{4}\left[1+\left(\Delta_{1}+\Delta_{2}\right)\right], \lambda_{5}^{B E}=\frac{1}{4}\left[1+\left(\Delta_{1}-\Delta_{2}\right)\right], \lambda_{6}^{B E}=$ $\frac{1}{4}\left[1-\left(\Delta_{1}+\Delta_{2}\right)\right]$, and $\lambda_{7}^{B E}=\frac{1}{4}\left[1-\left(\Delta_{1}-\Delta_{2}\right)\right]$, where $\Delta_{1}=\sqrt{\left(c_{0}^{2} p_{0}+c_{1}^{2} q_{0}\right)^{2}+\left(c_{0}^{2} p_{1}+c_{1}^{2} q_{1}\right)^{2}+c_{0}^{2} c_{1}^{2}\left(s_{0}+r_{0}\right)^{2}}$

and $\Delta_{2}=\sqrt{c_{0}^{2} c_{1}^{2}\left(s_{0}-r_{0}\right)^{2}} \cdot 3$ Considering the concavity of von Neumann entropy [16], we can find $S\left(\rho^{B E}\right)=-\sum_{i} \lambda_{i}^{B E} \log _{2} \lambda_{i}^{B E}$ approaches its maximum when $r_{0}=s_{0}=q_{1}=p_{1}=0$. In this case, we have $\lambda_{4,5}^{B E}=$ $\frac{1}{4}\left[1+\left(c_{0}^{2} p_{0}+c_{1}^{2} q_{0}\right)\right]$ and $\lambda_{6,7}^{B E}=\frac{1}{4}\left[1-\left(c_{0}^{2} p_{0}+c_{1}^{2} q_{0}\right)\right] .{ }^{4}$

\footnotetext{
${ }^{2}$ First, we select a completely orthogonal basis $\left|E_{00}^{\prime}\right\rangle,\left|E_{01}^{\prime}\right\rangle$, $\left|E_{11}^{\prime}\right\rangle$, and $\left|E_{10}^{\prime}\right\rangle$ on the Hilbert space $\mathscr{H}^{E}$. Then we have $\left|E_{00}\right\rangle=\sum_{i j} a_{i j}\left|E_{i j}^{\prime}\right\rangle, \quad\left|E_{01}\right\rangle=\sum_{i j} b_{i j}\left|E_{i j}^{\prime}\right\rangle, \quad\left|E_{11}\right\rangle=\sum_{i j} f_{i j}\left|E_{i j}^{\prime}\right\rangle$, and $\left|E_{10}\right\rangle=\sum_{i j} g_{i j}\left|E_{i j}^{\prime}\right\rangle$, where $i, j \in\{0,1\}$, and $\left\langle E_{00} \mid E_{01}\right\rangle=$ $s_{0}+i s_{1}, \quad\left\langle E_{00} \mid E_{10}\right\rangle=-\left\langle E_{01} \mid E_{11}\right\rangle=u_{0}+i u_{1}, \quad\left\langle E_{00} \mid E_{11}\right\rangle=p_{0}+$ $i p_{1},\left\langle E_{11} \mid E_{10}\right\rangle=r_{0}+i r_{1}$ and $\left\langle E_{01} \mid E_{10}\right\rangle=q_{0}+i q_{1}$. After some tedious calculations, we can gain the eigenvalues of $\rho^{A B E}$ on the basis $|0\rangle_{A},|1\rangle_{A},|0\rangle_{B},|1\rangle_{B},\left|E_{00}^{\prime}\right\rangle,\left|E_{01}^{\prime}\right\rangle,\left|E_{11}^{\prime}\right\rangle$, and $\left|E_{10}^{\prime}\right\rangle$ in the Hilbert space $\mathscr{H}^{A B E}=\mathscr{H}^{A} \otimes \mathscr{H}^{B} \otimes \mathscr{H}^{E}$, and the eigenvalues of $\rho^{B E}$ on the basis $|0\rangle_{B},|1\rangle_{B},\left|E_{00}^{\prime}\right\rangle,\left|E_{01}^{\prime}\right\rangle,\left|E_{11}^{\prime}\right\rangle$, and $\left|E_{10}^{\prime}\right\rangle$ in the Hilbert space $\mathscr{H}^{B E}=\mathscr{H}^{B} \otimes \mathscr{H}^{E}$.

${ }^{3}$ Calculation of the eigenvalues of $\rho^{A B E}$ and $\rho^{B E}$ was partially performed by computers with symbolic computation. It is surprising at first glance that the parameters $u_{i}, v_{i}, s_{1}$, and $r_{1}$ are canceled out in the formula for the eigenvalues of $\rho^{B E}$. The reason that $u_{i}$ and $v_{i}$ are canceled out is that $u_{i}$ and $v_{i}$ are symmetric with $u_{i}=-v_{i}$. Also, $s_{1}$ and $r_{1}$ are canceled out because $s_{1}$ and $r_{1}$ are the imaginary parts of $\left\langle E_{00} \mid E_{01}\right\rangle$ and $\left\langle E_{11} \mid E_{10}\right\rangle$, respectively, and thus they can be canceled out by diagonalization as arbitrary phase factors. Similarly, the eigenvalues of $\rho^{A B E}$ are independent of the parameters $u_{i}, v_{i}, r_{i}$, $s_{i}, p_{i}$, and $q_{i}$.

${ }^{4}$ As discussed earlier, Eve has to attack the traveling qubit twice, on both the Bob-Alice and Alice-Bob channels, to gain Alice's key bits. On the Bob-Alice channel, Eve attacks the forward qubit to distinguish its states, and then she can determine Alice's encoding operations after she attacked the backward qubit on the Alice-Bob channel. It is optimal for Eve to distinguish Alice's states when $\left\langle E_{00} \mid E_{01}\right\rangle=\left\langle E_{00} \mid E_{10}\right\rangle=\left\langle E_{11} \mid E_{10}\right\rangle=\left\langle E_{01} \mid E_{11}\right\rangle=0$, $\left\langle E_{00} \mid E_{11}\right\rangle=p_{0}$, and $\left\langle E_{01} \mid E_{10}\right\rangle=q_{0}$ [21], and thus it is optimal among collective attacks in the four-state protocol.
}

With the eigenvalues of $\rho^{A B E}$ and $\rho^{B E}$, we get

$$
\begin{gathered}
S\left(\rho^{A B E}\right)=2, \\
S\left(\rho^{B E}\right)=-\sum_{i} \lambda_{i}^{B E} \log _{2} \lambda_{i}^{B E} .
\end{gathered}
$$

From Eqs. (1) and (2), we can get

$$
\begin{aligned}
& 2 c_{++}\left|E_{++}\right\rangle=c_{00}\left|E_{00}\right\rangle+c_{01}\left|E_{01}\right\rangle+c_{11}\left|E_{11}\right\rangle+c_{10}\left|E_{10}\right\rangle . \\
& 2 c_{+-}\left|E_{+-}\right\rangle=c_{00}\left|E_{00}\right\rangle-c_{01}\left|E_{01}\right\rangle-c_{11}\left|E_{11}\right\rangle+c_{10}\left|E_{10}\right\rangle, \\
& 2 c_{--}\left|E_{--}\right\rangle=c_{00}\left|E_{00}\right\rangle-c_{01}\left|E_{01}\right\rangle+c_{11}\left|E_{11}\right\rangle-c_{10}\left|E_{10}\right\rangle, \\
& 2 c_{-+}\left|E_{-+}\right\rangle=c_{00}\left|E_{00}\right\rangle+c_{01}\left|E_{01}\right\rangle-c_{11}\left|E_{11}\right\rangle-c_{10}\left|E_{10}\right\rangle .
\end{aligned}
$$

When considering $f_{+}=f_{-}$, the equations above give a crucial boundary condition,

$$
1+c_{0}^{2} p_{0}+c_{1}^{2} q_{0}=2 c_{++}^{2} .
$$

Let us analyze the maximum of $S\left(\rho^{B E}\right)$ when the fidelity, $c_{0}^{2}$ and $c_{++}^{2}$, were verified by Alice and Bob in their postprocessing.

With the boundary condition of Eq. (6) and $-1 \leqslant$ $p_{0}, q_{0} \leqslant 1$, after some tedious calculation, we obtain that $c_{0}^{2} p_{0}+c_{1}^{2} p_{1} \geqslant 2 c_{++}^{2}-1-2 c_{1}^{2},{ }^{5}$ and $S\left(\rho^{B E}\right)$ approaches the maximum when $c_{0}^{2} p_{0}+c_{1}^{2} p_{1}=2 c_{++}^{2}-1-2 c_{1}^{2} \geqslant 0$. After verifying the condition $c_{++}^{2}-c_{1}^{2} \geqslant 1 / 2$ in their postprocessing, Alice and Bob can obtain

$$
\begin{aligned}
S\left(\rho^{B E}\right)= & \max \left\{S\left(\rho^{B E} \mid p_{0}, q_{0}\right)\right\} \\
& =-\frac{2 c_{++}^{2}-2 c_{1}^{2}}{2} \log _{2} \frac{2 c_{++}^{2}-2 c_{1}^{2}}{4} \\
& -\frac{2-2 c_{++}^{2}+2 c_{1}^{2}}{2} \log _{2} \frac{2-2 c_{++}^{2}+2 c_{1}^{2}}{4} .
\end{aligned}
$$

Therefore, after verifying $c_{++}^{2}-c_{1}^{2} \geqslant 1 / 2$ Alice and Bob can get the rate of PA against collective attacks,

$$
r_{\mathrm{PA}}(\xi)=S\left(\rho^{A} \mid \rho^{B E}\right)=1-h(\xi),
$$

where $\quad \xi=c_{++}^{2}-c_{1}^{2}, \quad$ and $\quad h(x)=-x \log _{2} x-(1-$ $x) \log _{2}(1-x)$ is the binary Shannon entropy.

In particular, if Eve does not attack the forward qubits in the Bob-Alice channel, i.e., $f_{0}=f_{1}=f_{+}=f_{-}=1$, one can find that $r_{\mathrm{PA}}(\xi)=1$. This states that Eve cannot gain any information about Alice's key bits if she does not attack the travel qubit in the Bob-Alice channel first.

Consider the case that Eve measures each forward qubit in the Bob-Alice channel in the basis $|0\rangle,|1\rangle$. Alice and Bob can verify that $f_{0}=f_{1}=1$, and $f_{+}=f_{-}=1 / 2$. In this case, we have $r_{\mathrm{PA}}(\xi)=0$. On the other hand, Eve can also measure each forward qubit in the Bob-Alice channel in the basis $|+\rangle,|-\rangle$, which gives $f_{+}=f_{-}=1$ and $f_{0}=f_{1}=1 / 2$, and thus $r_{\mathrm{PA}}(\xi)=0$. That is, Eve can gain full information of

${ }^{5}$ With the boundary condition $1+c_{0}^{2} p_{0}+c_{1}^{2} q_{0}=2 c_{++}^{2}$, we can get $2 c_{++}^{2}-1-c_{0}^{2} p_{0}=c_{1}^{2} q_{0} \leqslant c_{1}^{2}\left|q_{0}\right| \leqslant c_{1}^{2}$, i.e., $c_{0}^{2} p_{0} \geqslant 2 c_{++}^{2}-1-$ $c_{1}^{2}$, since $\left|p_{0}\right| \leqslant 1$ and $\left|q_{0}\right| \leqslant 1$. Thus, we can find that $c_{0}^{2} p_{0}+$ $c_{1}^{2} q_{0} \geqslant c_{0}^{2} p_{0}-c_{1}^{2}\left|q_{0}\right| \geqslant c_{0}^{2} p_{0}-c_{1}^{2} \geqslant 2 c_{++}^{2}-1-2 c_{1}^{2}$. Here $2 c_{++}^{2}-$ $1-2 c_{1}^{2} \geqslant 0$, i.e., $c_{++}^{2}-c_{1}^{2} \geqslant 1 / 2$ is required and should be verified by Alice and Bob in their postprocessing. 
Alice's key bits if she has exactly known the forward states before Alice's encoding operations.

\section{E. PA for the practical quantum channels}

For the practical quantum channels, the condition $f_{0}=$ $f_{1}$ and $f_{+}=f_{-}$is too strict to be satisfied. We can use the following strategy to symmetrize Eve's channels and eliminate this condition. We first identify four locations: Alice's side and Bob's side of the Bob-Alice and Alice-Bob channels. For each bit, we randomly insert a bit flip operation $Y$ at these four locations. So the four locations are either all $Y$ or all $I$. In this way, the fidelities of the new Bob-Alice channel are simply the average of that of the original channel [i.e., the new $f_{0}$ and $f_{1}\left(f_{+}\right.$and $\left.f_{-}\right)$are the average of the old $f_{0}$ and $f_{1}\left(f_{+}\right.$and $\left.\left.f_{-}\right)\right] .{ }^{6}$ Thus, this justifies the conditions $f_{0}=f_{1}$ and $f_{+}=f_{-}$ used in the previous sections.

Now, let us simplify further. Consider the two $Y$ operations at Alice's side located before and after Alice's encoding operation. Since Alice's encoding performs either $Y$ or $I$, the two new $Y$ operations commute with Alice's encoding operation and cancel out. Now consider the two $Y$ operations at Bob's side, if he originally wants to send bit $b=0,1$ in basis $W=X, Z$ through the the Bob-Alice channel, he now sends bit $1-b$ in basis $W$ to implement $Y$. When he receives the qubit from the Alice-Bob channel, he performs $Y$ on the incoming qubit, measures in basis $W$, records the bit $b^{\prime}$, and computes $b \oplus b^{\prime}$ as Alice's key bit. This is the same as his measuring the incoming qubit in $W$, flipping the bit result $1-b^{\prime}$ to $b^{\prime}$, and computing $b \oplus b^{\prime}$ as Alice's key bit. Note that the input to the raw Bob-Alice channel is $1-b$ in the basis $W$ and the output of the raw Alice-Bob channel is measured in $W$ resulting in bit $1-b^{\prime}$. Thus, Bob can use this information directly to infer Alice's key bit as $(1-b) \oplus\left(1-b^{\prime}\right)=b \oplus b^{\prime}$ without actually implementing the two $Y$ operations. Since $b$ is uniformly distributed, so is $1-b$ and the original protocol is recovered (i.e., no need to introduce the extra four $Y$ operations) except that the averages of the fidelities (instead of the individual fidelities) determined in the check mode are used in the key generation formulas. Specifically, we can use $\xi=f_{+,-}+f_{0,1}-1$ in Eq. (8) to calculate PA for any quantum channels in the four-state protocol, where $f_{0,1}=\left(c_{00}^{2}+c_{11}^{2}\right) / 2$, $f_{+,-}=\left(c_{++}^{2}+c_{--}^{2}\right) / 2$, and $c_{i j}^{2}$ 's are the original fidelities of the Bob-Alice channel determined in the check mode.

\section{F. Final key generation rate}

In the postprocessing, Alice and Bob should estimate the fidelity in the Bob-Alice channel, and $e$ in the Alice-Bob channel. Then they will perform EC and PA to generate the final key bits. In an asymptotic scenario, after verifying

$$
f_{+,-}+f_{0,1} \geqslant 3 / 2 \text {, }
$$

\footnotetext{
${ }^{6}$ Essentially, the Bob-Alice channel in Eqs. (1) and (2) is symmetrized by $U_{B E}|\psi\rangle_{B}|E\rangle|0\rangle_{B^{\prime}}+Y_{B} U_{B E} Y_{B}|\psi\rangle_{B}|E\rangle|1\rangle_{B^{\prime}}$ where system $B^{\prime}$ indicates whether we have a bit flip or not. We can assume that we give $B^{\prime}$ to Eve after the qubit transmission so that the pure-state analysis in the previous sections still applies. Note that giving $B^{\prime}$ to Eve afterward means that $U_{B E}$ and system $E$ are independent of our extra bit flip.
}

Alice and Bob can obtain the secure final key against collective attacks with the generation rate

$$
r=1-h(\xi)-h(e),
$$

where $h(e)$ is the amount of key bits Alice and Bob should sacrifice in the EC. To compare the key rate performance of the two-way DQKD protocol given by Eq. (10) to that of the BB84 protocol given by $1-2 h(e)$, we can assume a symmetric attack by Eve in the DQKD protocol case (in which $c_{++}^{2}=1-e$ and $c_{1}^{2}=e$ ) so that $\xi=1-2 e$. Thus, the key rate of the DQKD protocol is $1-h(2 e)-h(e)$, which is smaller than that of the $\mathrm{BB} 84$ protocol when there are errors.

\section{G. Security against general attacks}

Our analysis above assumes that the state $\rho^{A B E}$ of each run of the system is independent of and identical to the states of other runs, i.e., we assume the entire state for the $n$ runs is $\left(\rho^{A B E}\right)^{\otimes n}$. This collective-attack result can be extended to general attacks where the entire state is arbitrary without any restriction on the $n$ subsystems. Note that the four-state protocol is unchanged with a randomized permutation step. In light of Refs. [18,19], after permuting the $n$ subsystems and discarding $k$ of them, the resulting $n-k$ subsystems can be approximated by $n-k$ independent and identically distributed subsystems. ${ }^{7}$ Therefore, the final key bits in our proof with the generation rate in Eq.(10) are secure against general attacks.

\section{CONCLUSION}

We have proved that the four-state protocol is secure against general attacks, thus ending the long-standing dispute about the security of the deterministic QKD protocol. Our work may be extended to other QKD protocols with a two-way quantum channel and shine new light on the universality of QKD.

\section{ACKNOWLEDGMENTS}

We are grateful to Marco Lucamarini and Baocheng Zhang for their helpful discussions. Q.Y.C. especially thanks Hoi-Kwong Lo for his hospitality when visiting the University of Toronto. Financial support from RGC Grants No. HKU 701007P and No. 700709P of the HKSAR government and NSFC Grant No. 11074283 is gratefully acknowledged.

\footnotetext{
${ }^{7}$ The quantum de Finetti theorem applies only to finite dimensional Hilbert spaces. In our proof, Eve's state is a purification of Alice and Bob's state. Since the latter is finite dimensional, the former is also finite dimensional. Thus, Alice and Bob's knowledge about Eve's state is finite dimensional, despite that Eve's knowledge about her own state could still be infinite dimensional. This can be seen by the finite number of eigenvalues of the joint $A B E$ state given in the paragraph below Eq. (5). Also, the Stinespring dilation theorem states that Eve's effective ancilla is no more than four dimensional since the dimensionality of the Hilbert spaces of a travel qubit is 2 (see Sec. III B). Therefore, the application of the quantum de Finetti theorem is valid in our proof.
} 
[1] C. H. Bennett and G. Brassard, in Proceedings of the IEEE International Conference on Computers, Systems, and Signal Processing, Bangalore, India (IEEE, New York, 1984), p. 175.

[2] D. Mayer, J. ACM 48, 351 (2001).

[3] H.-K. Lo and H. F. Chau, Science 283, 2050 (1999).

[4] P. W. Shor and J. Preskill, Phys. Rev. Lett. 85, 441 (2000).

[5] V. Scarani, H. Bechmann-Pasquinucci, N. J. Cerf, M. Dušek, N. Lütkenhaus, and M. Peev, Rev. Mod. Phys. 81, 1301 (2009), and references therein.

[6] K. Boström and T. Felbinger, Phys. Rev. Lett. 89, 187902 (2002).

[7] A. Wójcik, Phys. Rev. Lett. 90, 157901 (2003); Q.-Y. Cai, ibid. 91, 109801 (2003).

[8] Q.-Y. Cai and B.-W. Li, Phys. Rev. A 69, 054301 (2004).

[9] Q.-Y. Cai and B.-W. Li, Chin. Phys. Lett. 21, 601 (2004).

[10] F.-G. Deng and G. L. Long, Phys. Rev. A 69, 052319 (2004).

[11] M. Lucamarini and S. Mancini, Phys. Rev. Lett. 94, 140501 (2005).

[12] K. Boström and T. Felbinger, Phys. Lett. A 372, 3953 (2008).
[13] Z.-J. Zhang, Z.-X. Man, and Y. Li, Phys. Lett. A 333, 46 (2004). [14] Z.-J. Zhang, Y. Li, and Z.-X. Man, Phys. Lett. A 341, 385 (2005). [15] Q.-Y. Cai, Phys. Lett. A 351, 23 (2006).

[16] M. A. Nielsen and I. L. Chuang, Quantum Computation and Quantum Information (Cambridge University Press, Cambridge, UK, 2000).

[17] C. M. Caves, C. A. Fuchs, and R. Schack, J. Math. Phys. 43, 4537 (2002); R. König and R. Renner, ibid. 46, 122108 (2005).

[18] R. Renner, Nat. Phys. 3, 645 (2007).

[19] M. Christandl, R. König, and R. Renner, Phys. Rev. Lett. 102, 020504 (2009).

[20] R. Renner and R. König, in Theory of Cryptography: Second Theory of Cryptography Conference, TCC 2005, Cambridge, MA, USA, February 10-12, 2005, Proceedings, Lecture Notes in Computer Science / Security and Cryptology, Vol. 3378, edited by J. Kilian (Springer, New York, 2005), p. 407.

[21] N. Gisin, G. Ribordy, W. Tittel, and H. Zbinden, Rev. Mod. Phys. 74, 145 (2002). 